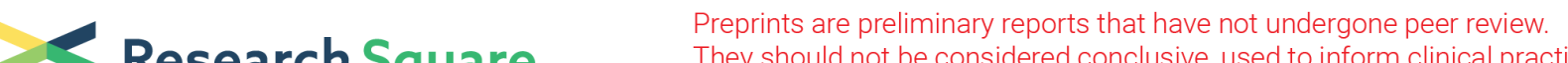 Research Square
or referenced by the media as validated information.
}

\section{Engrafted glial progenitor cells yield long-term integration and sensory improvement in aged mice}

\section{Zhiqi Yang}

Lanzhou University Second Hospital

\section{Mingyue Gong}

Third Military Medical Univeristy

\section{Tingliang Jian}

Third Military Medical University: Army Medical University

Jin Li

Third Military Medical University

\section{Chuanyan Yang}

Third Military Medical University

\section{Qinlong Ma}

Third Military Medical University

\section{Ping Deng}

Third Military Medical University

\section{Yuxia Wang}

Third Military Medical University

\section{Mingzhu Huang}

Third Military Medical University

\section{Haoyu Wang}

Third Military Medical University: Army Medical University

\section{Shaofan Yang}

Third Military Medical University: Army Medical University

\section{Xiaowei Chen}

Third Military Medical University: Army Medical University

\section{Zhengping Yu}

Third Military Medical University: Army Medical University

\section{Manxia Wang}

Lanzhou University Second Hospital

\section{Chunhai Chen}

Third Military Medical University: Army Medical University

Kuan Zhang ( $\nabla$ zhangkuan@tmmu.edu.cn )

Third Military Medical University: Army Medical University 


\section{Short report}

Keywords: glial progenitor cells, transplantation, endfeet, aquaporin-4, sensory response, aging

Posted Date: November 8th, 2021

DOI: https://doi.org/10.21203/rs.3.rs-1046233/v1

License: (c) (i) This work is licensed under a Creative Commons Attribution 4.0 International License. Read Full License 


\section{Abstract}

Aging causes astrocyte morphological degeneration and functional deficiency, which impairs neuronal functions. Until now, whether age-induced neuronal deficiency could be alleviated by engraftment of glial progenitor cell (GPC) derived astrocytes remained unknown. In the current study, GPCs were generated from embryonic cortical neural stem cells in vitro and transplanted into the brains of aged mice. Their integration and intervention effects in the aged brain were examined 12 months after transplantation. Results indicated that these in-vitro-generated GPC-derived astrocytes possessed normal functional properties. After transplantation they could migrate, differentiate, achieve long-term integration, and maintain much younger morphology in the aged brain. Additionally, these GPC-derived astrocytes established endfeet expressing aquaporin-4 (AQP4) and ameliorate AQP4 polarization in the aged neocortex. More importantly, age-dependent sensory response degeneration was reversed by GPC transplantation. This work demonstrates that rejuvenation of the astrocyte niche is a promising treatment to prevent age-induced degradation of neuronal and behavioral functions.

\section{Introduction}

Aging produces numerous detrimental changes in the brain including mitochondrial dysfunction, oxidative stress, and chronic inflammation ${ }^{[1]}$. These changes subsequently induce morphological degeneration and functional deficiency of astrocytes. It was demonstrated that aged astrocytes undergo morphological atrophy which reflects a decrease in their territorial domains and perisynaptic processes ${ }^{2}$, 3]. Age-induced astroglial atrophy results in trimming of synaptic contacts which impairs neurotransmitter clearance and synaptic plasticity ${ }^{[3,4]}$, and decreases endfeet coverage of brain vessels, thus contributing to deficits in the neurogliovascular unit ${ }^{[5]}$. Recent studies also showed that aged astrocytes create an inflammatory microenvironment permissive to synapse elimination and neuronal damage, leading to ageassociated cognitive decline ${ }^{[1,6]}$.

Glial progenitor cells (GPCs) arise from neural stem cells and exhibit context-dependent differentiation as astrocytes and oligodendrocytes ${ }^{[7,8]}$. As we reviewed previously ${ }^{[9]}$, the utility of GPCs in cell therapy has been reported in a variety of neurological diseases resulting from glial disorders, including demyelination disease ${ }^{[10]}$, amyotrophic lateral sclerosis (ALS) ${ }^{[11]}$, stroke ${ }^{[12]}$, and Alzheimer's Disease (AD) ${ }^{[13]}$. However, whether engrafted GPCs are able to yield effective intervention in brain aging remains unclear. To our knowledge, no previous study has systematically assessed the ability of GPCs to migrate, differentiate, and integrate within aged brain tissue or improve impaired behavior induced by cerebral dysfunction in aged animals.

In prior studies, fetal GPCs and pluripotent stem cells-derived GPCs were used as the cell resources for cell-transplant therapy ${ }^{[10,12,14,15]}$. However, both of these have limitations in further clinical applications, due to the initial finite number of fetal GPCs and the tumorigenesis of pluripotent stem cell-derived GPCs ${ }^{[8]}$. Given these complications, we investigated the differentiation and integration of GPCs derived from 
embryonic neural stem cells (NSCs) within the host brain in our previous work ${ }^{[16]}$. We found that these GPCs not only exhibited molecular and morphological features of mature astrocytes, but also functionally integrated into the cerebral sensory circuits of the host animals ${ }^{[16]}$.

Here, we examined the morphological and functional integration of GPCs derived from embryonic NSCs in aged mouse brains. We found that these GPCs were able to migrate, differentiate, achieve long-term integration, and remain much younger morphologically in the aged brain. More importantly, these engrafted GPC-derived astrocytes reversed the depolarization of perivascular aquaporin-4 (AQP4) and age-dependent sensory function degeneration in aged animals. The current study unveils transplantation of GPCs derived from embryonic NSCs as an effective strategy to ameliorate age-induced changes in the host brain via functional rejuvenation of aged neural circuits.

\section{Materials And Methods}

Detailed methods are shown in the Supplemental Information.

\section{Experimental Design}

Cortical NSCs were obtained from the embryonic brains 14.5-15.5 day old EGFP / PC-G5-tdT:Aldh111Cre/ERT2 transgenic mice. GPCs were in vitro generated from these embryonic cortical NSCs and transplanted into the primary somatosensory cortex (S1) of adult mice (6-8 months old). 12 months after transplantation the migration, differentiation, and long-term integration of engrafted GPCs were evaluated in the aged brains; the sensory functions of aged mice were also assessed (Supplementary Fig. 1A).

\section{Results}

Glial progenitor cells (GPCs) were generated in vitro and possess functional properties of primary astrocytes

Glial progenitor cells (GPCs) comprise an already lineage-restricted glial progenitor population, that may be more appropriate for treatment of glial disorders ${ }^{[17]}$. However, it is difficult to instruct in vivo differentiation of neural stem cells (NSCs) to GPCs ${ }^{[18]}$. Hence, in order to obtain a stable source of GPCs for the following transplantations, we previously developed a high-efficiency in vitro protocol for generating GPCs from embryonic cortical NSCs ${ }^{[16]}$ (Supplementary Fig. 1A). According to this protocol, GPCs were generated from NSCs and used for the following transplantation experiments (Supplementary Fig. 1B-D). Further experiments confirmed that these GPCs acquired the astrocytic differentiation potential (Supplementary Fig. 1E and F).

Astrocytic $\mathrm{Ca}^{2+}$ transients relate to a wide variety of significant functions ${ }^{[19,20]}$. To determine if in vitro generated GPC-derived astrocytes possess these $\mathrm{Ca}^{2+}$ events, we crossed the Cre-dependent GCaMP5G 
mouse line, termed PC-G5-tdT (Polr2a, CAG, GCaMP5G, tdTomato) ${ }^{[21]}$, with the Aldh1/1-Cre/ERT2 mouse line ${ }^{[22]}$, to obtain a line that expresses the GCaMP5G genetically-encoded $\mathrm{Ca}^{2+}$ indicator specifically in astrocytes (Fig. 1A ${ }^{[23]}$. It has been shown that following treatment with tamoxifen, almost all in-vitrogenerated GPC-derived astrocytes, identified as GFAP positive cells, were labeled by expression of both GCaMP5G and tdTomato (Fig. 1B). To investigate the functionality of these in-vitro-generated GPCderived astrocytes, we directly activated the astrocytes via focal application of adenosinetriphosphate (ATP), a P2Y agonist known to induce $\mathrm{Ca}^{2+}$ release from the internal stores of primary astrocytes ${ }^{[24]}$. Focal ATP $(200 \mu \mathrm{mol} / \mathrm{L})$ administration evoked a cytosolic $\mathrm{Ca}^{2+}$ increase in astrocytes that propagated across the field of view as a wave (Fig. $1 \mathrm{C}$ ). This propagation of $\mathrm{Ca}^{2+}$ waves across astrocytes plays a critical role in glial and neuron-glial cell communication ${ }^{[25]}$. The mean ATP-evoked peak $\Delta F / F_{0}$ was 185.0 $\pm 13.8 \%$ ( $n=50$ cells, Fig. 1D, E). Therefore, similar to primary astrocytes, astrocytes derived from in vitro generated GPCs possess $\mathrm{Ca}^{2+}$ transients and are competent for network communication.

\section{Engrafted GPCs differentiate into astrocytes with younger morphology and maintain long-term integration in the aged neocortex}

In our previous study, we found that engrafted GPCs could morphologically and functionally integrate into the adult mammalian neocortex ${ }^{[16]}$. However, it was not clear whether the engrafted GPCs could migrate, differentiate, and maintain long-term integration in the aged mammalian neocortex. To explore these processes, in-vitro-generated GPCs were transplanted into the somatosensory cortex of 6-8 month old mice, which were sacrificed 12 months after transplantation for histological analysis (Supplementary Fig. 1A).

The dispersal pattern of donor cells is a critical indicator of their integration in the host brain ${ }^{[14,26]}$. Our data revealed that 12 months after transplantation the engrafted GPCs had migrated widely in the somatosensory cortex and no signs of tumor formation were observed. They advanced into both the superficial and layers of the cortex (Fig. 2A). Furthermore, the vast majority of engrafted GPCs differentiated into astrocytes with complex star-like morphology and dense processes (Fig. 2B, C).

It was demonstrated that astrocytes display age-dependent morphological changes, including significant reductions in the number and the length of processes, territorial domains, and astrocyte-to-astrocyte coupling in the aged brain ${ }^{[2]}$. We next examined whether age-dependent structural degeneration would take place in engrafted astrocytes 12 months after transplantation. Consistent with previous studies ${ }^{[1,2,}$ 4], our data showed that cortical astrocytes of aged-control mice had a flattened shape, reductions in cellular surface area, and morphological complexity compared with those of adult-control ones (Fig. 2D, E, G, J-L). However, 12 months after transplantation the engrafted GPC-derived astrocytes in aged mice remained much younger morphologically and displayed more complex structure compared with the endogenous cortical astrocytes of aged-control mice (Fig. 2E, F). Statistical analysis also indicated that the engrafted GPC-derived astrocytes had more intersections (Fig. 2G, J, K) and primary branches (Fig. 2L). The engrafted GPC-derived astrocytes were also positive for connexin 30 (CX30) (Fig. 2H), a 
major astrocytic gap junction protein ${ }^{[27]}$, and D-serine (Fig. 2I), a gliotransmitter ${ }^{[28]}$. These findings indicated that engrafted GPC-derived astrocytes could form dynamic networks and regulate synaptic plasticity in the same manner as younger cells in the aged neocortex, 12 months after transplantation. These results demonstrate that engrafted GPCs are able to migrate, differentiate, retain a younger morphology, and achieve long-term integration in the aged mammalian brain.

\section{Engrafted GPC-derived astrocytes establish endfeet expressing AQP4 and reverse the depolarization of perivascular AQP4 in the aged neocortex}

Ageing causes degeneration of astrocytic endfeet ${ }^{[29]}$ and depolarization of perivascular AQP4 [30], resulting in prominent neurovascular dysfunction ${ }^{[29]}$ and the accumulation of protein waste ${ }^{[30]}$. Our previous studies demonstrated that engrafted astrocytes could establish endfeet along blood vessel walls ${ }^{[16]}$. However, it was unknown if the endfeet of engrafted GPC-derived astrocytes would be retained for a long time and express AQP4 in the aged brain. Our histological results revealed that extended endfeet (white arrows, Fig. 3A) from engrafted GPC-derived astrocytes still contiguously arrayed along the vessel wall (outlined with dashes, Fig. 3A, right panel) 12 months after transplantation in the aged brain. Additionally, AQP4 as expressed and remained on the endfeet (white arrows, Fig. 3B). More interestingly, our results revealed that engrafted GPC-derived astrocytes ameliorated AQP4 polarization in the aged mouse cortex (Fig. 3C-E). AQP4 localization became dispersed in the cortex of aged-control mice brains but remained highly polarized in brain regions engrafted with GPC-derived astrocytes (Fig. 3C-E). Ameliorated AQP4 polarization in the aged brain facilitates the clearance of interstitial solutes and contributes to the improvement of neuronal functions ${ }^{[31]}$.

\section{Engrafted Gpc-derived Astrocytes Reverse Age-induced Sensory Function Deficiency}

Our previous work revealed that engrafted GPC-derived astrocytes in the somatosensory cortex are able to respond to sensory stimulation with $\mathrm{Ca}^{2+}$ signals ${ }^{[16]}$. In addition, it has been reported that the somatosensory cortex experiences age-dependent morphological and functional degeneration ${ }^{[32-36]}$. We subsequently investigated whether the integration of engrafted GPC-derived astrocytes and their amelioration of AQP4 polarization could yield any potential functional improvement in the aged somatosensory cortex.

Previous studies indicated that the somatosensory cortex is involved in sensorimotor integration and sensory response modulation ${ }^{[37-39]}$. To assess the functional properties of this brain region, we examined the escape response latencies of the sensory response in aged GPC-transplanted mice 12 months post transplantation (Fig. 4A). Consistent with previous reports ${ }^{[33-36]}$, our study found obvious functional degeneration of the somatosensory cortex of aged-control mice which showed much longer escape response latencies, as compared with adult-control mice (Fig. 4B, C). In contrast, 12 months after transplantation of GCPs in the somatosensory cortex, engrafted aged mice showed an improved sensory 
response, exhibiting obviously reduced escape response latencies compared with the aged-control mice (Fig. 4B, C). Thus, the engrafted GPC-derived astrocytes not only achieved morphologically long-term integration and ameliorated AQP4 polarization in the aged somatosensory cortex, but also functionally reversed the age-dependent functional degeneration of this brain region.

\section{Discussion}

Aging is characterized by chronic, low-grade and systemic inflammation which leads to time-dependent deterioration in the brain ${ }^{[40]}$. During this process, astrocytes undergo morphological degeneration and functional impairment ${ }^{[41]}$. Astrocytic dysfunction significantly changes the microenvironment of the brain, resulting in increased oxidative damage and reduced metabolic activity of neurons and the inhibition of neuroprotective capabilities ${ }^{[42]}$. Here, we examined whether rejuvenating the astrocyte niche by transplantation of GPCs can improve the neuronal functioning of aged brains. It has found that engrafted GPCs can migrate, differentiate, achieve long-term integration, and ameliorate AQP4 polarization in the aged mammalian brain. This rejuvenation of the astrocyte niche was able to reverse the functional degeneration of neurons in the aged somatosensory cortex.

Aged astrocytes exhibit both morphological and functional remodeling with a predominance of morphological atrophy and functional loss ${ }^{[3]}$. The reduced size and complexity of astrocytes results in decreased astroglial synaptic coverage with subsequent decline in glutamate clearance, metabolic support, and synaptic plasticity ${ }^{[2,3]}$. Previous studies have reported that engrafted GPCs could differentiate and structurally integrate into host neural circuits of different adult mouse/rat disease models, including those used to study adult demyelination disease ${ }^{[43]}, \mathrm{ALS}^{[44]}$, stroke ${ }^{[12]}$ and Alzheimer's disease $(A D)^{[13]}$. Therefore, engrafted GPCs are ideal cell sources for generating new healthy young astrocytes which can replace senescent and dysfunctional astrocytes in the aged brain. Consistent with previous reports, our study showed that engrafted GPC-derived astrocytes yield long-term structural integration in the aged mouse brain. More interestingly, they displayed much younger morphology compared with the aged host's astrocytes. One possible explanation is that engrafted GPCs may maintain higher steady-state activity of antioxidant mechanisms ${ }^{[45]}$ and resist the hostile pathological microenvironment better than the native host cell populations ${ }^{[45]}$.

Aging induces decreased coverage of astrocyte endfeet on blood vessels, which impairs the astroglialvascular coupling and functions of the blood-brain barrier ${ }^{[3,41]}$. Additionally, aging is associated with impaired glymphatic clearance caused by the activation of astrocytes and depolarization of protein AQP4, resulting in the accumulation of protein waste and neuroinflammation ${ }^{[30]}$. Our results provide evidence that engrafted GPC-derived astrocytes can establish endfeet along blood vessel walls and these newly formed endfeet are able to express AQP4. Further results demonstrated that this rejuvenated astrocyte niche was able to ameliorate AQP4 polarization in the aged neocortex. Thus, engrafted GPCderived astrocytes may improve perivascular clearance and reduce neuroinflammation, thereby promoting the survival of nearby neurons in the aged brain. 
It has been reported that engrafted GPCs exhibit superior neuroprotective effects and improved behavioral outcomes in various adult mouse/rat disease models, including stroke ${ }^{[12]}$, Huntington's disease ${ }^{[46]}$, Parkinson's disease ${ }^{[47]}$ and demyelination disease ${ }^{[48]}$. In the present study, we also demonstrate that the morphologically younger engrafted GPC-derived astrocytes restored the effects of age-induced sensory function deficiency. This improved behavioral function in aged mice may be induced by the rejuvenation of the astrocyte niche ${ }^{[49]}$, resulting in faster glutamate clearance, more stable homeostasis in the CNS, and more efficient modulation of synaptic activity. All of these restored astrocytic functions create a healthier micro-environment for neuronal activity in the aged brain.

Taken together, our results indicate that rejuvenating the astrocyte niche can reverse age-induced sensory function degradation. This is the first study to demonstrate that age-related impairment of neuronal functions could be improved by the transplantation of GPC-derived astrocytes. In conclusion, the present study indicates that the introduction of astrocytes, the main support cells of the central nervous system, is a promising potential treatment for preventing age-induced degradation of neuronal and behavioral functions.

\section{Abbreivations}

GPC: glial progenitor cell; aquaporin-4 (AQP4); ALS: amyotrophic lateral sclerosis; AD: Alzheimer's Disease; NSCs: neural stem cells; S1: somatosensory cortex; ATP: adenosinetriphosphate;

\section{Declarations}

\section{Acknowledgements}

We thank Jia Lou for technical assistance.

\section{Authors' contributions}

This work was designed by C.C. and K.Z.. The main experiments were performed by Z.Y., M.G., T.J., J.L., C.Y., Q.M., P.D., Y.W., M.H., H.W., S.Y., X.C., Z.Y., M.W. C.C. and K.Z.. Z.Y., M.G., Q.M. and P.D. conducted stem cell maintenance and differentiation. In vitro $\mathrm{Ca}^{2+}$ imaging was performed by Y.Z.. Y.Z., J.L., C.Y. H.W., S.F. and K.Z.. conducted cell transplantation. Z.Y., T.J., Y.W. and K.Z. performedimmunohistochemistry and confocal imaging. Behavioral test was performed by Z.Y.. The data analysis was performed by Z.Y., M.H., H.W., X.C., Z.Y., M.W., C.C. and K.Z. This manuscript was written by Z.Y., M.W., C.C. and K.Z. with input from all coauthors. All authors read and approved the final manuscript.

\section{Funding}

This work was supported by the National Key R \& D Program of China (2018YFA0109600), the National Natural Science Foundation of China (81771175) and the program of China Scholarship Council (201803170004). 
Availability of data and materials

The dataset used and analyzed during the current study are available from the corresponding author upon reasonable request.

\section{Ethics approval and consent to participate}

All animal experiments were carried out according to the guidelines (Reference Number: SCXK,20170002 and SYXK,20170002) approved by the Institutional Animal Care and Use Committee of the Third Military Medical University, China.

\section{Consent for publication}

Not applicable

\section{Competing interests}

The authors declare that they have no competing interests.

\section{Author details}

${ }^{1}$ Department of Neurology, Lanzhou University Second Hospital, Cuiyingmen 82, Chengguan District, Lanzhou 730030, Gansu, China. ${ }^{2}$ Brain Research Center and State Key Laboratory of Trauma, Burns, and Combined Injury, Third Military Medical University, Chongqing 400038, China. ${ }^{3}$ Department of Occupational Health, Third Military Medical University, Chongqing 400038, China

\section{References}

1. Cohen J, Torres C. Astrocyte senescence: Evidence and significance. Aging Cell 2019, 18: e12937.

2. Popov A, Brazhe A, Denisov P, Sutyagina O, Li L, Lazareva N, et al. Astrocyte dystrophy in ageing brain parallels impaired synaptic plasticity. Aging Cell 2021, 20: e13334.

3. Verkhratsky A, Augusto-Oliveira M, Pivoriūnas A, Popov A, Brazhe A, Semyanov A. Astroglial asthenia and loss of function, rather than reactivity, contribute to the ageing of the brain. Pflugers Arch 2021, 473: 753-774.

4. Rodríguez-Arellano JJ, Parpura V, Zorec R, Verkhratsky A. Astrocytes in physiological aging and Alzheimer's disease. Neuroscience 2016, 323: 170-182.

5. Tarantini S, Tran CHT, Gordon GR, Ungvari Z, Csiszar A. Impaired neurovascular coupling in aging and Alzheimer's disease: Contribution of astrocyte dysfunction and endothelial impairment to cognitive decline. Exp Gerontol 2017, 94: 52-58.

6. Boisvert MM, Erikson GA, Shokhirev MN, Allen NJ. The Aging Astrocyte Transcriptome from Multiple Regions of the Mouse Brain. Cell Rep 2018, 22: 269-285.

7. Gage FH. Mammalian neural stem cells. Science 2000, 287: 1433-1438. 
8. Goldman SA. Progenitor cell-based treatment of glial disease. Prog Brain Res 2017, 231: 165-189.

9. Zhang K, Chen X. Sensory response in host and engrafted astrocytes of adult brain in Vivo. Glia 2017, 65: 1867-1884.

10. Wang S, Bates J, Li X, Schanz S, Chandler-Militello D, Levine C, et al. Human iPSC-derived oligodendrocyte progenitor cells can myelinate and rescue a mouse model of congenital hypomyelination. Cell Stem Cell 2013, 12: 252-264.

11. Lepore AC, Rauck B, Dejea C, Pardo AC, Rao MS, Rothstein JD, et al. Focal transplantation-based astrocyte replacement is neuroprotective in a model of motor neuron disease. Nat Neurosci 2008, 11: 1294-1301.

12. Jiang P, Chen C, Wang R, Chechneva OV, Chung SH, Rao MS, et al. hESC-derived Olig2+ progenitors generate a subtype of astroglia with protective effects against ischaemic brain injury. Nat Commun 2013, 4: 2196.

13. Esposito G, Sarnelli G, Capoccia E, Cirillo C, Pesce M, Lu J, et al. Autologous transplantation of intestine-isolated glia cells improves neuropathology and restores cognitive deficits in $\beta$ amyloidinduced neurodegeneration. Sci Rep 2016, 6: 22605.

14. Han $X$, Chen $M$, Wang F, Windrem M, Wang S, Shanz S, et al. Forebrain engraftment by human glial progenitor cells enhances synaptic plasticity and learning in adult mice. Cell Stem Cell 2013, 12: 342-353.

15. Kondo Y, Windrem MS, Zou L, Chandler-Militello D, Schanz SJ, Auvergne RM, et al. Human glial chimeric mice reveal astrocytic dependence of JC virus infection. J Clin Invest 2014, 124: 53235336.

16. Zhang K, Chen C, Yang Z, He W, Liao X, Ma Q, et al. Sensory Response of Transplanted Astrocytes in Adult Mammalian Cortex In Vivo. Cereb Cortex 2016, 26: 3690-3704.

17. Goldman SA, Nedergaard M, Windrem MS. Glial progenitor cell-based treatment and modeling of neurological disease. Science 2012, 338: 491-495.

18. Osorio MJ, Goldman SA. Glial progenitor cell-based treatment of the childhood leukodystrophies. Exp Neurol 2016, 283: 476-488.

19. Lim D, Semyanov A, Genazzani A, Verkhratsky A. Calcium signaling in neuroglia. Int Rev Cell Mol Biol 2021, 362: 1-53.

20. Haydon PG, Nedergaard M. How do astrocytes participate in neural plasticity? Cold Spring Harb Perspect Biol 2014, 7: a020438.

21. Gee JM, Smith NA, Fernandez FR, Economo MN, Brunert D, Rothermel M, et al. Imaging activity in neurons and glia with a Polr2a-based and cre-dependent GCaMP5G-IRES-tdTomato reporter mouse. Neuron 2014, 83: 1058-1072.

22. Hu NY, Chen YT, Wang Q, Jie W, Liu YS, You QL, et al. Expression Patterns of Inducible Cre Recombinase Driven by Differential Astrocyte-Specific Promoters in Transgenic Mouse Lines. Neurosci Bull 2020, 36: 530-544. 
23. Srinivasan R, Lu TY, Chai H, Xu J, Huang BS, Golshani P, et al. New Transgenic Mouse Lines for Selectively Targeting Astrocytes and Studying Calcium Signals in Astrocyte Processes In Situ and In Vivo. Neuron 2016, 92: 1181-1195.

24. Salter MW, Hicks JL. ATP causes release of intracellular Ca2+ via the phospholipase C beta/IP3 pathway in astrocytes from the dorsal spinal cord. J Neurosci 1995, 15: 2961-2971.

25. Scemes E, Giaume C. Astrocyte calcium waves: what they are and what they do. Glia 2006, 54: 716725.

26. Windrem MS, Schanz SJ, Morrow C, Munir J, Chandler-Militello D, Wang S, et al. A competitive advantage by neonatally engrafted human glial progenitors yields mice whose brains are chimeric for human glia. J Neurosci 2014, 34: 16153-16161.

27. Kunzelmann P, Schröder W, Traub O, Steinhäuser C, Dermietzel R, Willecke K. Late onset and increasing expression of the gap junction protein connexin30 in adult murine brain and long-term cultured astrocytes. Glia 1999, 25: 111-119.

28. Schell MJ, Molliver ME, Snyder SH. D-serine, an endogenous synaptic modulator: localization to astrocytes and glutamate-stimulated release. Proc Natl Acad Sci U S A 1995, 92: 3948-3952.

29. Duncombe J, Lennen RJ, Jansen MA, Marshall I, Wardlaw JM, Horsburgh K. Ageing causes prominent neurovascular dysfunction associated with loss of astrocytic contacts and gliosis. Neuropathol Appl Neurobiol 2017, 43: 477-491.

30. Kress BT, Iliff JJ, Xia M, Wang M, Wei HS, Zeppenfeld D, et al. Impairment of paravascular clearance pathways in the aging brain. Ann Neurol 2014, 76: 845-861.

31. Li G, He X, Li H, Wu Y, Guan Y, Liu S, et al. Overexpression of Slit2 improves function of the paravascular pathway in the aging mouse brain. Int J Mol Med 2018, 42: 1935-1944.

32. Calì C, Wawrzyniak M, Becker C, Maco B, Cantoni M, Jorstad A, et al. The effects of aging on neuropil structure in mouse somatosensory cortex-A 3D electron microscopy analysis of layer 1. PLoS One 2018, 13: e0198131.

33. David-Jürgens M, Churs L, Berkefeld T, Zepka RF, Dinse HR. Differential effects of aging on fore- and hindpaw maps of rat somatosensory cortex. PLoS One 2008, 3: e3399.

34. Kalisch T, Ragert P, Schwenkreis P, Dinse HR, Tegenthoff M. Impaired tactile acuity in old age is accompanied by enlarged hand representations in somatosensory cortex. Cereb Cortex 2009, 19: 1530-1538.

35. Coq JO, Xerri C. Sensorimotor experience modulates age-dependent alterations of the forepaw representation in the rat primary somatosensory cortex. Neuroscience 2001, 104: 705-715.

36. Liguz-Lecznar M, Lehner M, Kaliszewska A, Zakrzewska R, Sobolewska A, Kossut M. Altered glutamate/GABA equilibrium in aged mice cortex influences cortical plasticity. Brain Struct Funct 2015, 220: 1681-1693.

37. Huttunen J, Wikström H, Korvenoja A, Seppäläinen AM, Aronen H, Ilmoniemi RJ. Significance of the second somatosensory cortex in sensorimotor integration: enhancement of sensory responses during finger movements. Neuroreport 1996, 7: 1009-1012. 
38. Xerri C, Merzenich MM, Peterson BE, Jenkins W. Plasticity of primary somatosensory cortex paralleling sensorimotor skill recovery from stroke in adult monkeys. J Neurophysiol 1998, 79: 21192148.

39. Halley AC, Baldwin MKL, Cooke DF, Englund M, Krubitzer L. Distributed Motor Control of Limb Movements in Rat Motor and Somatosensory Cortex: The Sensorimotor Amalgam Revisited. Cereb Cortex 2020, 30: 6296-6312.

40. Matias I, Morgado J, Gomes FCA. Astrocyte Heterogeneity: Impact to Brain Aging and Disease. Front Aging Neurosci 2019, 11: 59.

41. Palmer AL, Ousman SS. Astrocytes and Aging. Front Aging Neurosci 2018, 10: 337.

42. Tang $Y$, Wang J, Lin $X$, Wang L, Shao B, Jin $K$, et al. Neural stem cell protects aged rat brain from ischemia-reperfusion injury through neurogenesis and angiogenesis. J Cereb Blood Flow Metab 2014, 34: 1138-1147.

43. Windrem MS, Schanz SJ, Zou L, Chandler-Militello D, Kuypers NJ, Nedergaard M, et al. Human Glial Progenitor Cells Effectively Remyelinate the Demyelinated Adult Brain. Cell Rep 2020, 31: 107658.

44. Chen H, Qian K, Chen W, Hu B, Blackbourn LWt, Du Z, et al. Human-derived neural progenitors functionally replace astrocytes in adult mice. J Clin Invest 2015, 125: 1033-1042.

45. Madhavan L, Ourednik V, Ourednik J. Increased "vigilance" of antioxidant mechanisms in neural stem cells potentiates their capability to resist oxidative stress. Stem Cells 2006, 24: 2110-2119.

46. Benraiss A, Wang S, Herrlinger S, Li X, Chandler-Militello D, Mauceri J, et al. Human glia can both induce and rescue aspects of disease phenotype in Huntington disease. Nat Commun 2016, 7: 11758.

47. L'Episcopo F, Tirolo C, Peruzzotti-Jametti L, Serapide MF, Testa N, Caniglia S, et al. Neural Stem Cell Grafts Promote Astroglia-Driven Neurorestoration in the Aged Parkinsonian Brain via Wnt/ $\beta$-Catenin Signaling. Stem Cells 2018, 36: 1179-1197.

48. Piao J, Major T, Auyeung G, Policarpio E, Menon J, Droms L, et al. Human embryonic stem cellderived oligodendrocyte progenitors remyelinate the brain and rescue behavioral deficits following radiation. Cell Stem Cell 2015, 16: 198-210.

49. Das MM, Avalos P, Suezaki P, Godoy M, Garcia L, Chang CD, et al. Human neural progenitors differentiate into astrocytes and protect motor neurons in aging rats. Exp Neurol 2016, 280: 41-49.

\section{Figures}


A

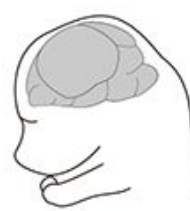

PC-G5-tdT

Aldh1l1-Cre/ERT2
Cultured in mdeium with

CNTF ( $10 \mathrm{ng} / \mathrm{mL}$ ), $30 \%$ FBS and

$4 \mathrm{OH}$ - tomaxifen $(1 \mu \mathrm{mol} / \mathrm{L})$

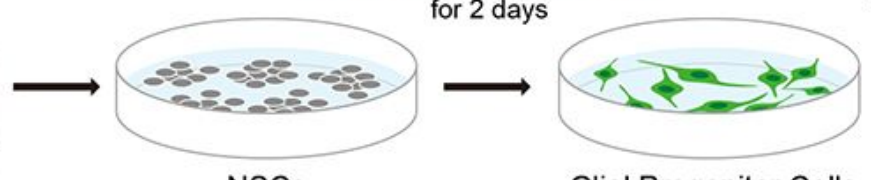

NSCs
Glial Progenitor Cells

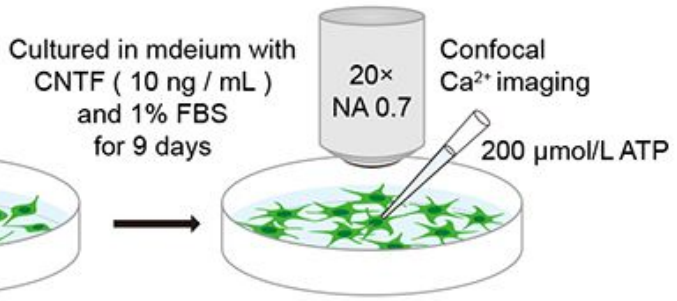

Astrocytes
B

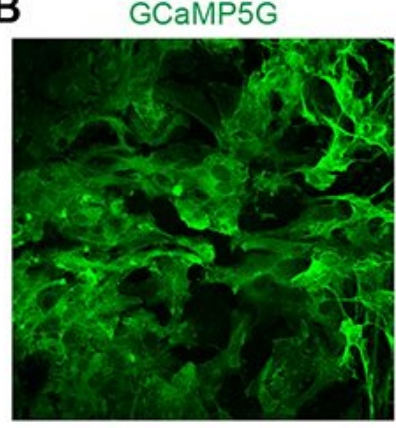

C
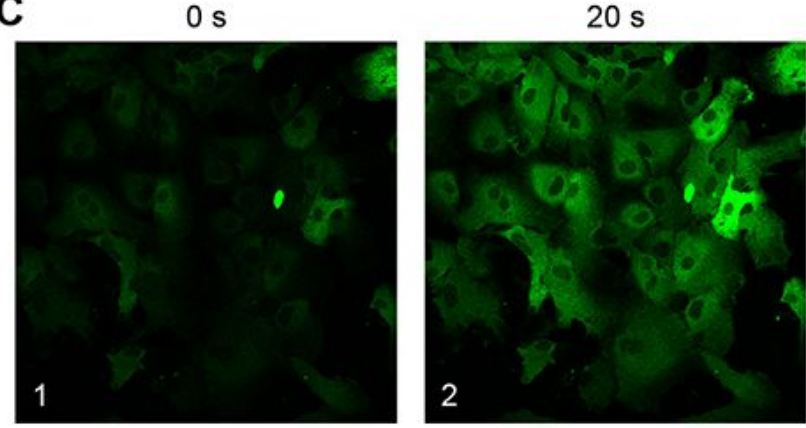

GFAP

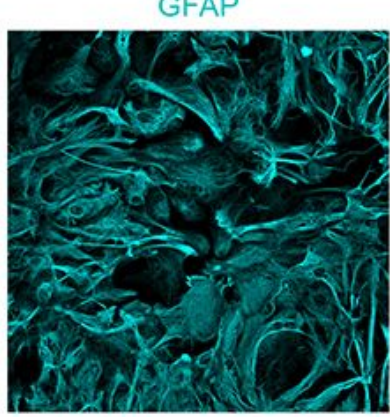

$70 \mathrm{~s}$

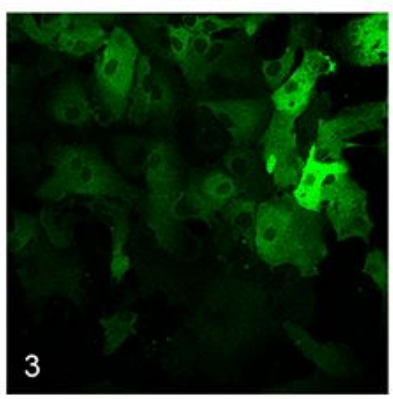

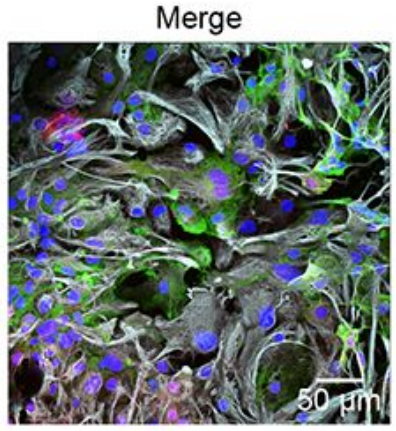

$140 \mathrm{~s}$

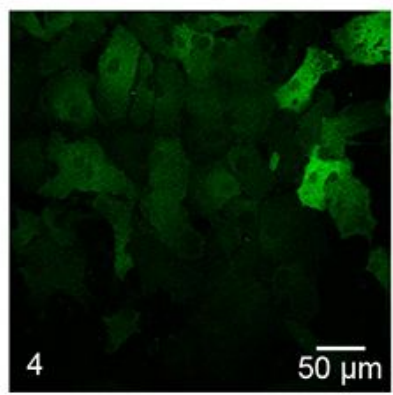

E

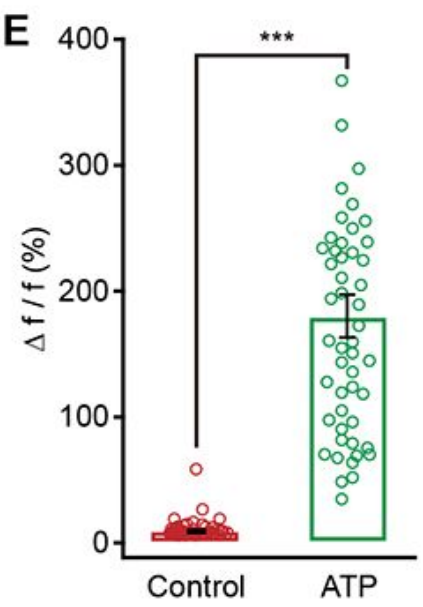

Contro

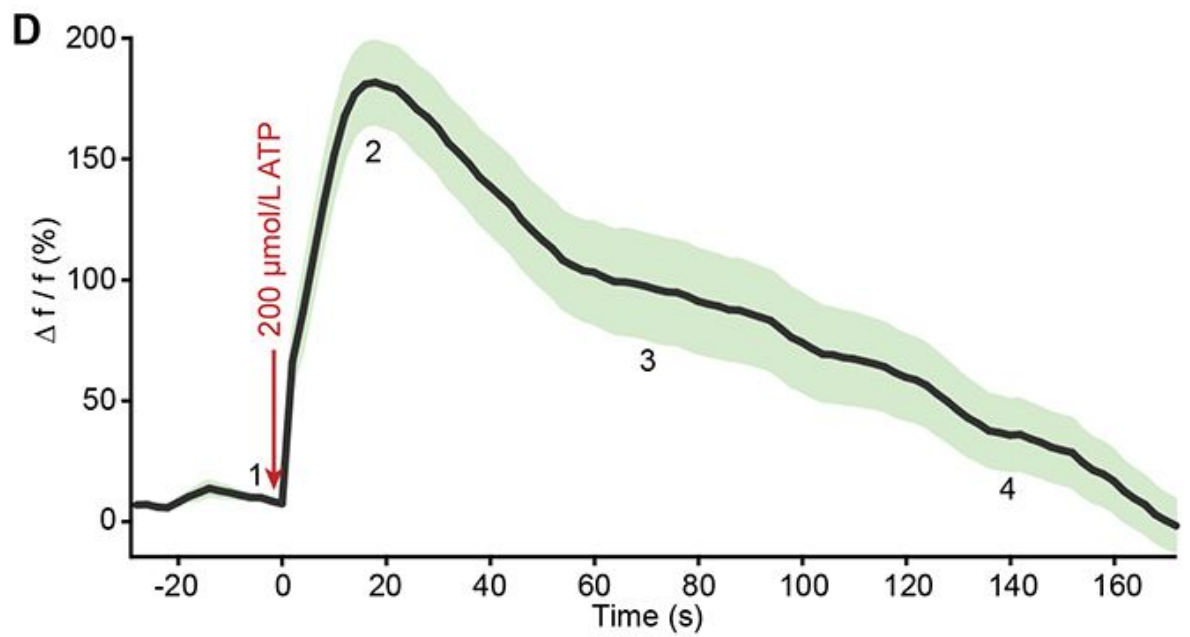

\section{Figure 1}

$\mathrm{Ca} 2+$ transients can be induced in cultured astrocytes generated from embryonic cortical NSCs. (A) Experimental procedure for NSC isolation, metamorphic recombination of Cre-ERT2, glial precursor cell induction, and confocal Ca2+ imaging. (B) Mature astrocytes (GFAP+, cyan) can be induced from NSCs and express both GCaMP5G (green) and tdTomato (red) following treatment with tamoxifen. (C) Ca2+ imaging of cultured astrocytes derived from NSCs labeled with GCaMP5G (green) at 4 time points after 
addition of ATP. (D) Ca2+ signals evoked by $200 \mu \mathrm{M}$ ATP in the derived astrocytes ( $\mathrm{n}=49$ cells). The 4 time points shown in panel $\mathrm{c}$ are labeled on the trace of $\mathrm{Ca} 2+$ signals. (E) Bar graphs of astrocytic $\mathrm{Ca} 2+$ amplitude $(\Delta f / f)$ without (control) or with ATP ( $n=49$ cells in each group; Control versus ATP, $Z=-6.903$, $\mathrm{P}=1.1101 \mathrm{E}-09 ; * \star * \mathrm{P}<0.001$, two-sided Wilcoxon signed-rank test). All data in the figure are shown as mean \pm s.e.m.

A
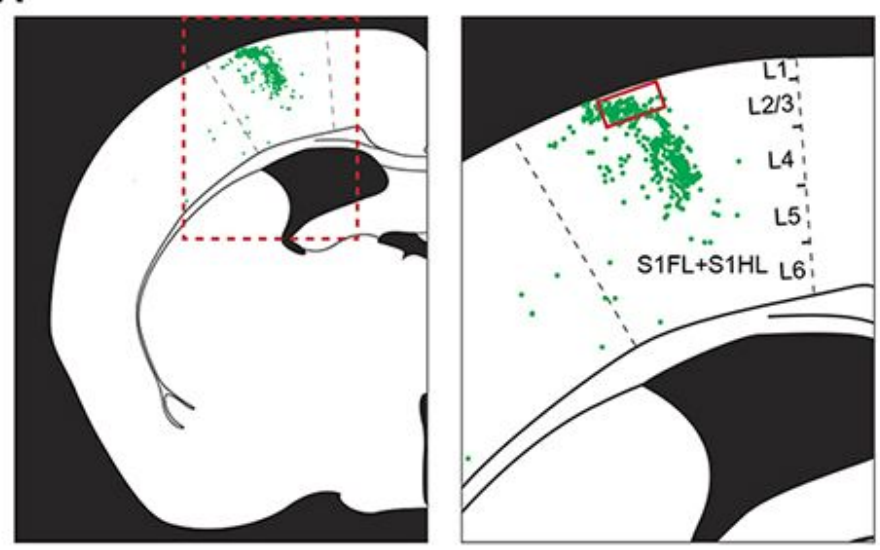

E Aged-control

D Adult-control GFAP

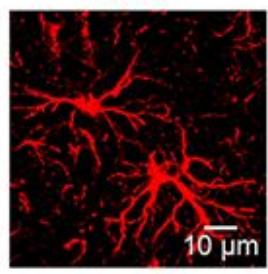

G
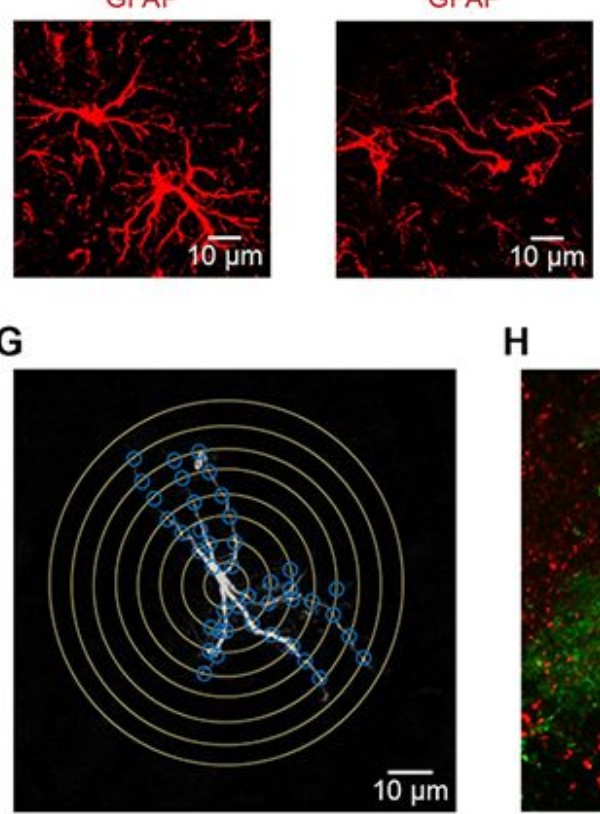

H
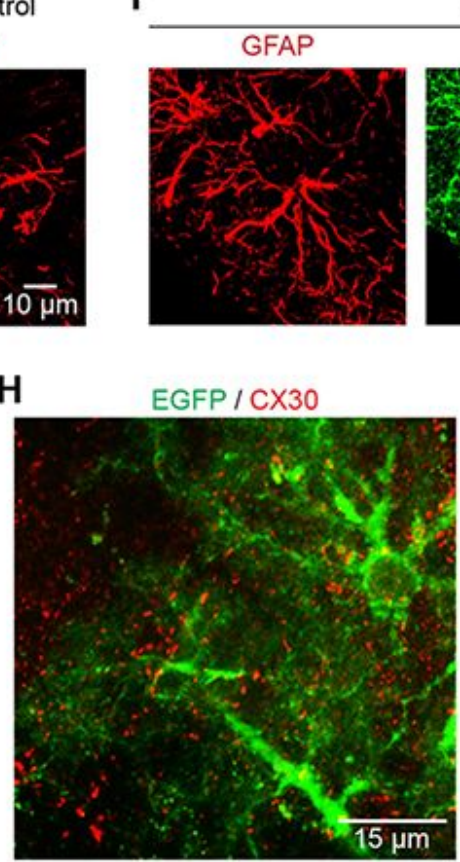

Aged-engrafted
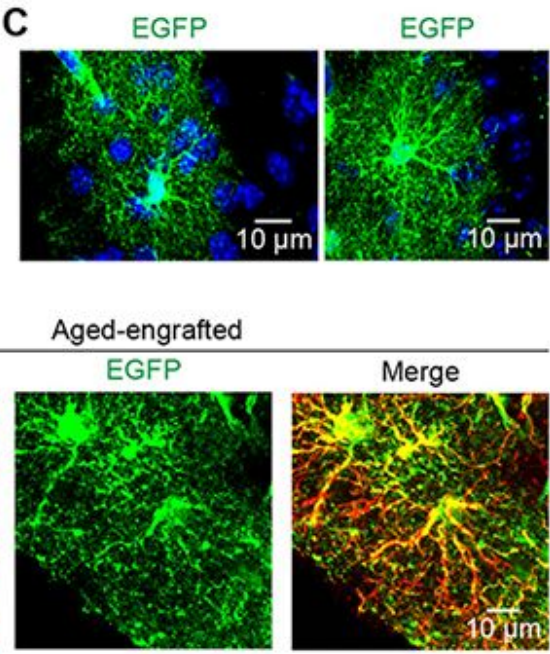

I
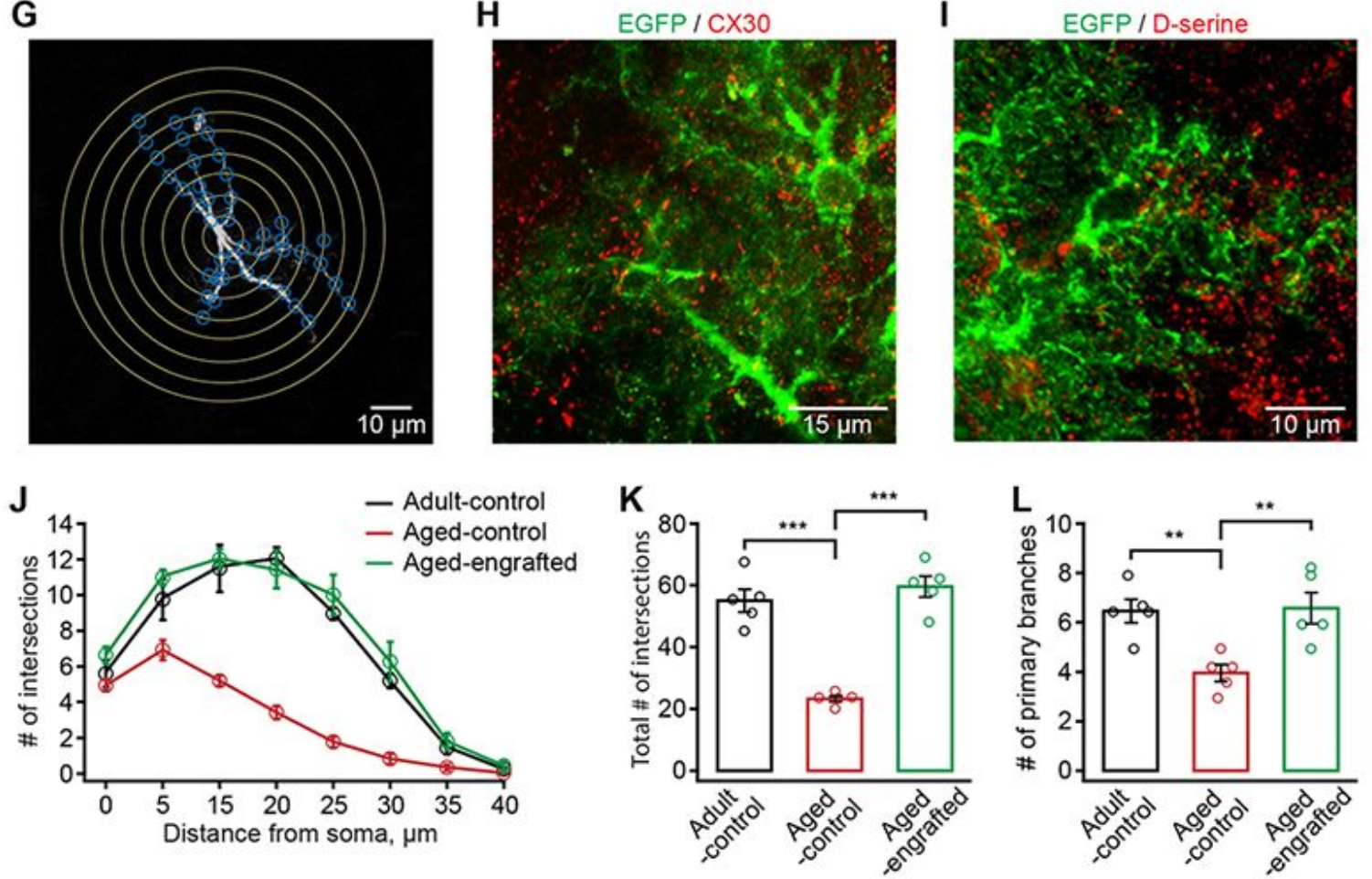

Figure 2 
Engrafted in-vitro-generated GPCs achieve long-term morphological integration and remain morphologically younger in the aged neocortex. (A) Representative dot map showing the distribution of engrafted GPC-derived astrocytes 12 months after transplantation (left panel, the coronal section of the half brain; right panel, primary somatosensory cortex outlined by the red dashed line in the left panel. S1FL: primary somatosensory cortex, forelimb region; S1HL: primary somatosensory cortex, hindlimb region.). Engrafted GPC-derived astrocytes were distributed in different cortical layers in the somatosensory cortex. (B-C) Representative images of engrafted GPC-derived astrocytes, positive for EGFP, 12 months after transplantation (B, the network of astrocytes outlined by the red line in the right panel of A. C, Higher magnification of single astrocytes showing the complex fine structures of engrafted GPC-derived astrocytes). (D-F) Representative confocal 3-dimensional reconstructed images showing GFAP-immunoreactive astrocytes in adult-control (D), aged-control, (E) and aged-engrafted mice groups (F). Engrafted astrocytes are also labeled with EGFP protein (F). (G) Sholl analysis for the measurement of the relative number of astrocyte processes. The morphology of an astrocyte was traced and outlined from the GFAP labeling (white). Concentric rings (yellow) were placed $5 \mu \mathrm{m}$ apart around the cell. Branching points, where astrocytic processes made intersections (blue) with a concentric ring, were used to quantify the relative number of processes. $(\mathrm{H}-\mathrm{I})$ Engrafted GPC-derived astrocytes express a gap junction protein $(\mathrm{H})$, connexin 30 (CX30), and a gliotransmitter, D-serine (I) 12 months after transplantation. $(\mathrm{J})$ Single astrocyte Sholl analysis showing the number of intersections of astrocytic branches and branchlets with concentric spheres centered in the middle of cell soma $(n=5$ mice per group). (K-L) Summary of total the intersection number (K) and primary branches number (L) in adultcontrol, aged-control, and aged-engrafted mice groups ( $n=5$ mice per group; total number of intersections: adult-control versus aged-control, $P=1.90 \mathrm{E}-5$; aged-control versus aged-engrafted, $P=4.29 \mathrm{E}-6$; number of primary branches: adult-control versus aged-control, $P=0.0116$; aged-control versus aged-engrafted, $\mathrm{P}=0.0086 ;{ }^{* \star} \mathrm{P}<0.01,{ }^{\star} * \star \mathrm{P}<0.001$, two-way ANOVA with Bonferroni post hoc comparisons test). All data in the figure are shown as mean \pm s.e.m.. 
A

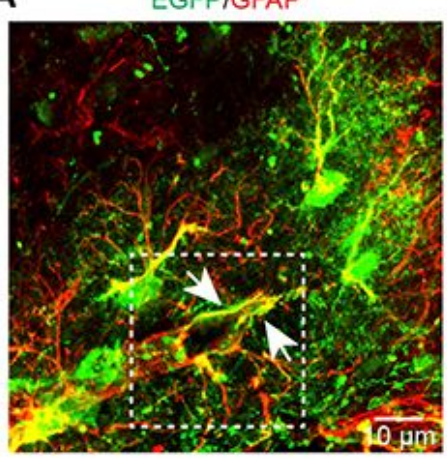

C

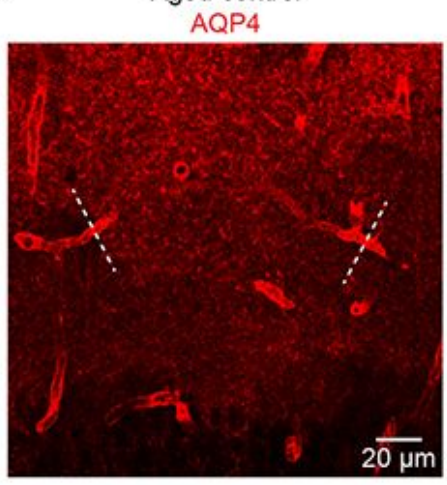

EGFPIGFAP

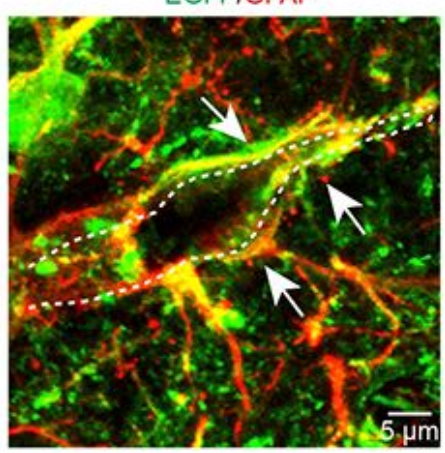

Aged-engrafed EGFPIAQP4

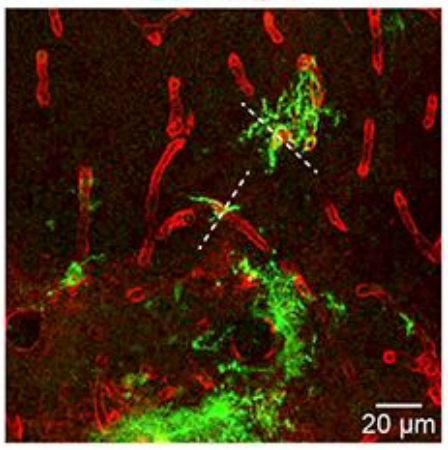

B

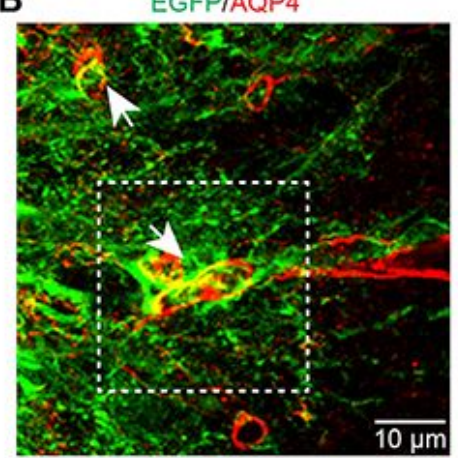

D

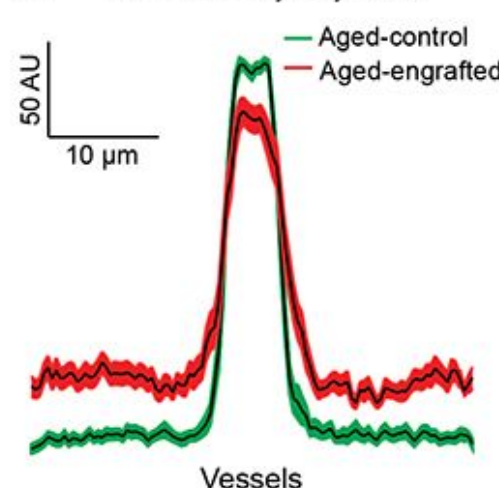

EGFPIAQP4

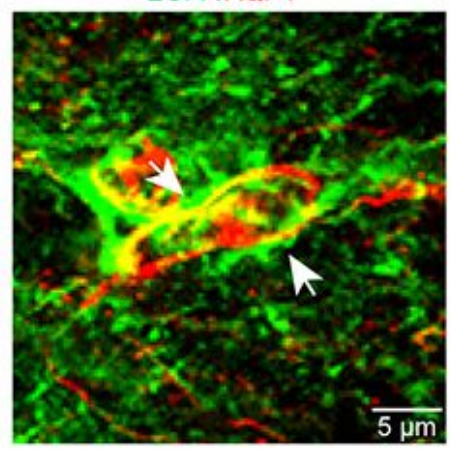

E

E Perivascular AQP4

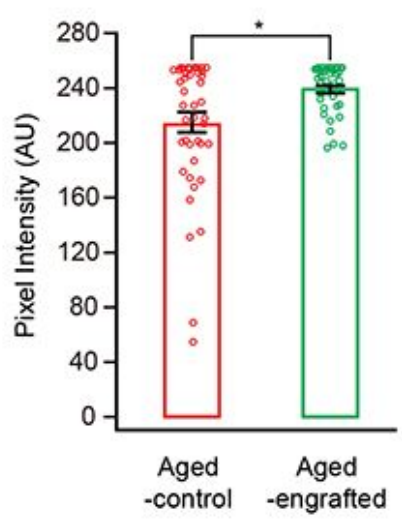

\section{Figure 3}

Engrafted GPC-derived astrocytes (EGFP+) establish endfeet expressing AQP4 and ameliorate AQP4 polarization in the aged neocortex. (A) Endfeet of engrafted GPC-derived astrocytes arrayed along the blood vessels 12 months after transplantation in the aged neocortex (white arrows in left panel). Higher magnification (outlined by the white dashed box in the left panel) showing endfeet of engrafted GPCderived astrocytes (white arrows in right panel) wrapping the vessel wall. (B) Expression of AQP4 (white arrows) distributed on the endfeet of engrafted GPC-derived astrocytes (EGFP+) 12 months after transplantation in the aged neocortex. Higher magnification (outlined by the white dashed box in the left panel) showing the expressions of AQP4 (white arrows) remained on the endfoot membranes of engrafted GPC-derived astrocytes (EGFP+). (C) Perivascular AQP4 polarization was lost in the neocortex of aged-control brain (left panel) but remained in the cortex region transplanted with GPC-derived astrocytes (EGFP+, right panel) of the aged-engrafted brain. (D) AQP4 immunofluorescence evaluated in linear regions of interest (dashed lines, C) extending outward from vessels. (E) Bar graph summarizing measurement of perivascular AQP4 expression. Compared with the aged-control brain, perivascular AQP4 expression was increased in surrounding blood vessels of the aged-engrafted brain ( $n=44$ vessels from 4 aged-control mice, $n=41$ vessels from 4 aged-engrafted mice; aged-control versus aged-engrafted, $Z=-2.202, P=0.028 ; * P<0.05$, two-sided Wilcoxon rank sum test). All data in the figure are shown as mean \pm s.e.m. 

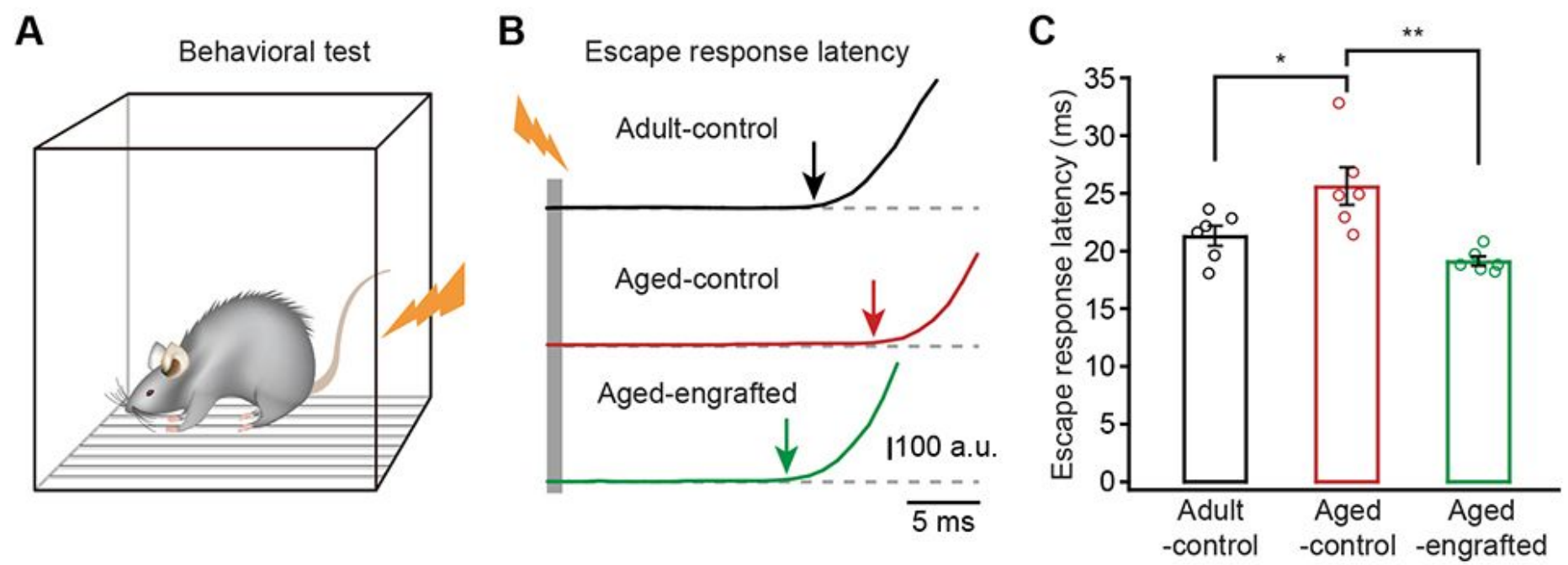

Figure 4

Sensory functions of aged mice are improved by engraftment of GPC-derived astrocytes in the somatosensory cortex. (A) Schematic illustration of the experimental protocol used for testing escape response latency. (B) Response traces of mice after footshock stimulation (grey bar) in adult-control, aged-control, and aged-engrafted mice groups. (C) Summary of escape response latencies in adultcontrol, aged-control, and aged engrafted mice group ( $\mathrm{n}=6$ mice per group; Adult-control versus Agedcontrol, $P=0.0401$; Aged-control versus Aged-engrafted, $P=0.0022 ; * P<0.05, * \star P<0.01$, two-way ANOVA with Bonferroni post hoc comparisons test). All data in the figure are shown as mean \pm s.e.m..

\section{Supplementary Files}

This is a list of supplementary files associated with this preprint. Click to download.

- SupplementalFig.1.docx

- Supplementalinformation.docx 